\title{
Seabed geoacoustic characterization with a vector sensor array $^{\text {a) }}$
}

\author{
P. Santos, O. C. Rodríguez, P. Felisberto, and S. M. Jesus ${ }^{\text {b) }}$ \\ Institute for Systems and Robotics, University of Algarve, Campus de Gambelas, \\ PT8005-139 Faro, Portugal
}

(Received 9 December 2009; revised 11 June 2010; accepted 11 August 2010)

\begin{abstract}
This paper proposes a vector sensor measurement model and the related Bartlett estimator based on particle velocity measurements for generic parameter estimation, illustrating the advantages of the Vector Sensor Array (VSA). A reliable estimate of the seabed properties such as sediment compressional speed, density and compressional attenuation based on matched-field inversion (MFI) techniques can be achieved using a small aperture VSA. It is shown that VSAs improve the resolution of seabed parameter estimation when compared with pressure sensor arrays with the same number of sensors. The data considered herein was acquired by a four-element VSA in the $8-14 \mathrm{kHz}$ band, during the Makai Experiment in 2005. The results obtained with the MFI technique are compared with those obtained with a method proposed by C. Harrison, which determines the bottom reflection loss as the ratio between the upward and downward beam responses. The results show a good agreement and are in line with the historical information for the area. The particle velocity information provided by the VSA increases significantly the resolution of seabed parameter estimation and in some cases reliable results are obtained using only the vertical component of the particle velocity. (C) 2010 Acoustical Society of America. [DOI: 10.1121/1.3488305]
\end{abstract}

PACS number(s): 43.30.Pc, 43.60.Kx, 43.60.Jn, 43.60.Fg [AIT] Pages: 2652-2663

\section{INTRODUCTION}

Acoustic vector sensors measure both the acoustic pressure and the three components of particle velocity. Each vector sensor has four channels, one for the omni-directional pressure sensor and three for the particle velocity-meters which are sensitive only in a specific direction. In the last decade the interest in vector sensors increased exponentially, influenced by electromagnetic vector sensor applications and developments in sensor technology that allowed building compact arrays for acoustic applications in the air. It is expected that in the near future vector sensors will be commercially available for developing compact underwater Vector Sensor Arrays (VSA) at a reasonable cost. The VSA has advantage in direction of arrival (DOA) estimation when compared with traditional pressure sensor arrays. Its potential can be extended to other underwater applications. The main objective of this work is to illustrate the advantages of VSA over pressure sensor arrays for high-frequency (8-14 $\mathrm{kHz}$ ) seabed geoacoustic parameter estimation (sediment compressional speed, density and compressional attenuation).

During the last decade, several authors conducted research on the theoretical aspects of vector sensors processing, suggesting that this type of device has advantages in DOA estimation and gives rise to an improved resolution. ${ }^{1-8}$ Nehorai and Paldi ${ }^{1}$ extended an analytical model, initially

\footnotetext{
${ }^{a}$ Part of this work was presented at the Third International Conference on Underwater Acoustic Measurements: Technologies and Results, Nafplion, Greece, June 2009.

b) Author to whom correspondence should be addressed. Electronic mail: pjsantos@ualg.pt
}

developed for electromagnetic sources, to the underwater acoustic case. The comparison of the DOA estimation performance of a VSA and that of an array of pressure sensors shows the advantages of the VSA. The authors also derived a compact expression for the Cramér-Rao Lower Bound (CRLB) on the estimation errors of the source DOA. Cray and Nuttall ${ }^{2}$ showed that the VSA has an increased directivity gain not achieved by hydrophone arrays of the same length. Tabrikian et $a l^{3}$ proposed an efficient electromagnetic vector sensor configuration for source localization in the air and analyzed the CRLB. The authors have found that the minimum number of sensors, capable of estimating the DOA of an arbitrary polarized signal from any direction, is two electric and two magnetic sensors referred to as a quadrature vector sensor. Wan et al. ${ }^{4}$ performed comparative simulation studies of the DOA estimation using classic methods such as MUSIC and MVDR with VSAs, gradient sensors and pressure sensors. The results shows that pressure and vector arrays outperform gradient hydrophone arrays, that consist of three pressure hydrophone symmetrically mounted in a circle. The applications of the VSA can also be found in port and waterway security, underwater communications and geoacoustic inversion. This type of sensor has long been desired by the Navy to provide directional information on target noise sources. Shipps and Abraham ${ }^{5}$ described the new vector sensor developed for the Navy as particularly useful in underwater acoustic surveillance and port security. Lindwall $^{6}$ showed the advantage of using vector data over scalar data for image structures in a 3-D volume verified by an experiment using a vector sensor in a water tank. Recently, theoretical work ${ }^{7,8}$ using quaternion based algorithms is proposed to more effectively process VSA data for DOA 
estimation. In Refs. 9 and 10 the feasibility of using vector sensors in underwater acoustic communications was investigated. The results suggest that vector sensors can offer an attractive acoustic communication solution for compact underwater platforms and underwater autonomous vehicles, where space is very limited. The high directivity and the ability of VSA to provide directional information can also be used in geoacoustic inversion. Peng and $\mathrm{Li}^{11}$ propose a geoacoustic inversion scheme based on experimental data measured by a VSA at low frequency (central frequency $400 \mathrm{~Hz}$ ), where it was shown that the VSA can reduce the uncertainty in the estimation of the sediment compressional speed.

Matched-field techniques in underwater acoustics were introduced by Hinich, ${ }^{12}$ who has used the spatial complexity of the underwater acoustic field to propose a new source localization method. This concept was developed and adapted to geoacoustic and tomography inversion - matchedfield inversion (MFI). The estimation of the seabed geoacoustic parameters can be posed as an optimization problem using techniques, such as genetic algorithms ${ }^{13}$ or simulated annealing ${ }^{14}$ to address a large number of parameters over a wide search space, traditionally made using pressure sensor arrays. These techniques can be implemented, in principle, with particle velocity information. The objective of this paper is not to propose an operational optimization technique but to understand the potential gain of combining particle velocity sensors with pressure sensors, for estimating seabed parameters with standard estimation techniques. The main focus of this work is the application of the Bartlett estimator with measured high-frequency VSA data to estimate seabed geoacoustic parameters. This paper presents a vector sensor measurement model and the related Bartlett estimator theory based on particle velocity for generic parameter estimation. The proposed geoacoustic inversion methods based on MFI techniques show the advantage of including particle velocity information to improve the resolution of the estimated parameters. Some of these parameters are difficult to estimate with pressure sensors alone, even with large aperture arrays. An existing Gaussian beam model was specifically adapted to generate field replicas which include both the acoustic pressure and the particle velocity outputs. The experimental data considered herein was acquired by a four element vertical VSA in the $8-14 \mathrm{kHz}$ band, during the Makai experiment, off Kauai I., Hawaii (USA) from 15 September to 2 October 2005. ${ }^{15}$ Previous work with this data included the DOA estimation using a plane wave beamformer. ${ }^{16}$ In this work, the measured high-frequency VSA data is used for seabed geoacoustic parameters estimation such as the sediment compressional speed, density and compressional attenuation and the results are of considerable interest due to their uniqueness in this research area. The frequency band used is well above that traditionally used in geoacoustic inversion. Preliminary results on the estimation of the sediment compressional speed were presented in. ${ }^{17}$ The results are consistent with previous measurements in the area and indicate that, when particle velocity is included, it can significantly improve the resolution of seabed geoacoustic parameter estimation. In some cases, such improvement is obtained using only the vertical component of the particle velocity.

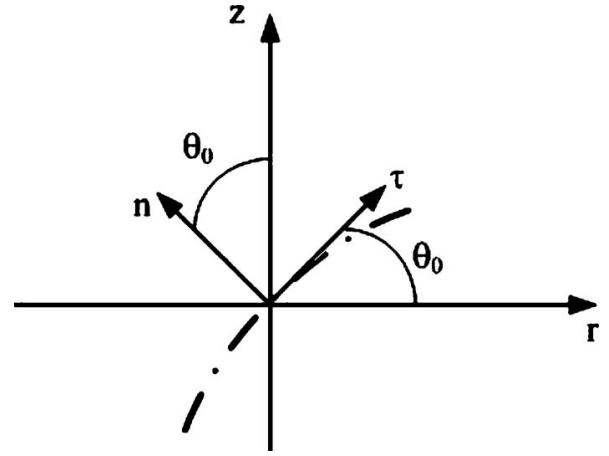

FIG. 1. Diagram of the ray trajectory (dashdot line) with ray unitary vectors $\boldsymbol{\tau}$ and $\mathbf{n}$ projections onto the horizontal $r$ and vertical $z$ axes.

This work suggests that a VSA of only a few elements provides a sufficiently compact setup to be embarked on reduced dimension autonomous moving platforms as an alternative to existing bottom profilers.

This paper is organized as follows: Section II describes the vector sensor measurement model and the theory related to the Bartlett estimator based on particle velocity for generic parameter estimation; Section III presents the simulation results of DOA and seabed parameters estimation comparing the acoustic pressure with the particle velocity results; Section IV considers the inversion of the seabed parameters with the data acquired by a VSA during the MakaiEx 2005 using two methods: 1) by forward modeling of reflection loss and data comparison and 2) by MFI based on the Bartlett estimator; and finally Section V presents the conclusions of this work.

\section{THE VECTOR SENSOR DATA MODEL}

This section presents a comprehensive data model which incorporates both pressure and particle velocity sensors. The signal model is derived by adapting the existing Gaussian beam model to also provide the particle velocity.

\section{A. Modeling particle velocity using Gaussian beams}

Let consider the general geometry of the tangent $(\boldsymbol{\tau})$ and the normal $(\boldsymbol{n})$ ray unitary vectors, at a particular point of a ray trajectory, as shown by the dashdot line in Fig. 1.

Particle velocity (v) can be calculated from the linear acoustic equation (Euler's equation) through the relationship with the acoustic pressure as:

$$
\mathbf{v}=-\frac{i}{\omega \rho} \nabla p,
$$

where $\rho$ represents the density of the watercolumn and $\omega$ is the working frequency of the propagating acoustic wave. The pressure gradient $\nabla p$ at a particular point of the ray trajectory can be expressed as:

$$
\nabla p=\left[\frac{\partial p}{\partial s}, \frac{\partial p}{\partial N}\right],
$$

where $\partial p / \partial s$ and $\partial p / \partial N$ stand for the derivative along $\tau$ and $\boldsymbol{n}$, respectively, $N$ is the normal distance from the ray, $s$ is the 
arclength along the ray and the ray unitary vectors at that point are given by:

$$
\boldsymbol{\tau}=\left[\cos \theta_{0}, \sin \theta_{0}\right] \text { and } \boldsymbol{n}=\left[-\sin \theta_{0}, \cos \theta_{0}\right],
$$

where $\theta_{0}$ is the angle between the $(r, z)$ axes and the ray unitary vectors.

Hence, the horizontal and vertical particle velocity components $\left(v_{r}, v_{z}\right)$ are obtained by projecting the pressure gradient onto the $(r, z)$ axes as:

$$
\begin{aligned}
v_{r}= & \frac{\partial p}{\partial s} \cos \theta_{0}-\frac{\partial p}{\partial N} \sin \theta_{0} \text { and } v_{z}=\frac{\partial p}{\partial s} \sin \theta_{0} \\
& +\frac{\partial p}{\partial N} \cos \theta_{0} .
\end{aligned}
$$

While the VSA has three components the $v_{x}$ and $v_{y}$ components are calculated by projecting the horizontal particle velocity in the azimuthal direction of the source $\left(\varphi_{S}\right)$, previously estimated, then:

$$
v_{x}=v_{r} \cos \left(\varphi_{S}\right) \text { and } v_{y}=v_{r} \sin \left(\varphi_{S}\right) .
$$

Using the Gaussian beam approximation of the ray pressure given by ${ }^{18,19}$

$$
p(s, N)=P_{0}(s) \exp \left[-i \omega\left(\frac{s}{c(s)}+\frac{1}{2} \gamma(s) N^{2}\right)\right],
$$

where $c(s)$ is the sound speed at position $s, P_{0}(s)$ is an arbitrary constant and $\gamma(s)$ is related to beamwidth and curvature. $^{19}$

The analytical expressions for the pressure gradient components corresponds to:

$$
\frac{\partial p}{\partial s}=-i \frac{\omega}{c} p \quad \text { and } \quad \frac{\partial p}{\partial N}=-i \omega \gamma(s) N p .
$$

Assuming a small aperture array and a generic set of environmental parameters $\left(\Theta_{0}\right)$ that characterize the channel, including ocean bottom parameters, the particle velocity can be written as:

$$
\mathbf{v}\left(\Theta_{0}\right)=\mathbf{u}\left(\Theta_{0}\right) p,
$$

where

$$
\mathbf{u}\left(\Theta_{0}\right)=\left[\begin{array}{l}
u_{x}\left(\Theta_{0}\right) \\
u_{y}\left(\Theta_{0}\right) \\
u_{z}\left(\Theta_{0}\right)
\end{array}\right]=\left[\begin{array}{c}
i \omega \gamma(s) N \sin \theta_{0} \cos \varphi_{S}-i \frac{\omega}{c} \cos \theta_{0} \cos \varphi_{S} \\
i \omega \gamma(s) N \sin \theta_{0} \sin \varphi_{S}-i \frac{\omega}{c} \cos \theta_{0} \sin \varphi_{S} \\
-i \omega \gamma(s) N \cos \theta_{0}-i \frac{\omega}{c} \sin \theta_{0}
\end{array}\right],
$$

is the vector defined for a ray trajectory $\left(\theta_{0}\right)$. In a real scenario, not only one ray but several rays impinge the array. In this case $\mathbf{u}\left(\Theta_{0}\right)$ in Eq. (8) is defined as a sum of the contributions of each ray.

\section{B. Data model}

Assuming that the propagation channel is a linear timeinvariant system, $p$ is the acoustic pressure and $v_{x}, v_{y}$ and $v_{z}$ are the three particle velocity components, then the field measured at the vector sensor due to a source signal $s(t)$ is given by:

$$
y_{k}\left(t, \Theta_{0}\right)=h_{k}\left(t, \Theta_{0}\right) * s(t)+n_{k}(t),
$$

where $*$ denotes convolution, $\Theta_{0}$ is a parameter vector, $h_{k}\left(\Theta_{0}\right)$ is the channel impulse response and $n_{k}(t)$ is the additive noise for pressure and the three components of particle velocity for $k=p, v_{x}, v_{y}, v_{z}$, respectively.

Assuming a narrowband signal, the sensor output at a frequency $\omega$ (omitting the frequency dependency in the following) for a particular set of channel parameters $\Theta_{0}$ can be rewritten as:

$$
y_{k}\left(\Theta_{0}\right)=h_{k}\left(\Theta_{0}\right) s+n_{k},
$$

where $s$ is the source component at frequency $\omega, h_{k}\left(\Theta_{0}\right)$ is the channel response and $n_{k}$ is the additive noise.

Taking into account Eq. (8) and (9), the vector sensor model can be obtained as:

$$
\left[\begin{array}{c}
y_{p}\left(\Theta_{0}\right) \\
y_{v_{x}}\left(\Theta_{0}\right) \\
y_{v_{y}}\left(\Theta_{0}\right) \\
y_{v_{z}}\left(\Theta_{0}\right)
\end{array}\right]=\left[\begin{array}{c}
h_{p}\left(\Theta_{0}\right) \\
u_{x}\left(\Theta_{0}\right) h_{p}\left(\Theta_{0}\right) \\
u_{y}\left(\Theta_{0}\right) h_{p}\left(\Theta_{0}\right) \\
u_{z}\left(\Theta_{0}\right) h_{p}\left(\Theta_{0}\right)
\end{array}\right] s+\left[\begin{array}{c}
n_{p} \\
n_{v_{x}} \\
n_{v_{y}} \\
n_{v_{z}}
\end{array}\right] .
$$

In the following it is assumed that the additive noise is zero mean, white, both in time and space, ${ }^{20}$ with variance $\sigma_{n}^{2}$ and uncorrelated with the signal $s$, itself with zero mean and variance $\sigma_{s}^{2}$.

For an array of $L$ vector sensors, the acoustic pressure at frequency $\omega$ is given by:

$$
\mathbf{y}_{p}\left(\Theta_{0}\right)=\left[y_{p_{1}}\left(\Theta_{0}\right), \cdots, y_{p_{L}}\left(\Theta_{0}\right)\right]^{T},
$$

where $y_{p_{l}}\left(\Theta_{0}\right)$ is the acoustic pressure at the lth vector sensor. The linear data model for the acoustic pressure is:

$$
\mathbf{y}_{p}\left(\Theta_{0}\right)=\mathbf{h}_{p}\left(\Theta_{0}\right) s+\mathbf{n}_{p},
$$

where $\mathbf{h}_{p}\left(\Theta_{0}\right)$ is the channel frequency response at $L$ pressure sensors and $\mathbf{n}_{p}$ is the additive acoustic pressure noise.

A similar definition has been adopted for the particle velocity, where the velocity part of the measurement is

$$
\begin{aligned}
\mathbf{y}_{v}\left(\Theta_{0}\right)= & {\left[y_{v_{x}}\left(\Theta_{0}\right), \cdots, y_{v_{x}}\left(\Theta_{0}\right), y_{v_{y} 1}\left(\Theta_{0}\right), \cdots,\right.} \\
& \left.y_{v_{y} L}\left(\Theta_{0}\right), y_{v_{z} 1}\left(\Theta_{0}\right), \cdots, y_{v_{z} L}\left(\Theta_{0}\right)\right]^{T} .
\end{aligned}
$$

Considering short arrays, $\mathbf{u}\left(\Theta_{0}\right)$ is assumed to be approximately constant for all elements thus, the data model for the particle velocity components is given by:

$$
\mathbf{y}_{v}\left(\Theta_{0}\right)=\mathbf{u}\left(\Theta_{0}\right) \otimes \mathbf{h}_{p}\left(\Theta_{0}\right) s+\mathbf{n}_{v}
$$

where $\mathbf{n}_{v}$ is the additive noise satisfying the above assumptions and $\otimes$ is the Kronecker product. For $\mathbf{h}_{p}\left(\Theta_{0}\right)$ with dimension $L \times 1, \mathbf{y}_{v}\left(\Theta_{0}\right)$ has dimension $3 L \times 1$.

Santos et al:: Seabed characterization with a vector sensor array 
Combining Eq. (14) and (16) a complete VSA data model, formed by the acoustic pressure and the particle velocity, can be defined for a signal measured on $L$ vector sensor elements as:

$$
\mathbf{y}_{p v}\left(\Theta_{0}\right)=\left[\begin{array}{l}
\mathbf{y}_{p}\left(\Theta_{0}\right) \\
\mathbf{y}_{v}\left(\Theta_{0}\right)
\end{array}\right]=\left[\begin{array}{c}
1 \\
\mathbf{u}\left(\Theta_{0}\right)
\end{array}\right] \otimes \mathbf{h}_{p}\left(\Theta_{0}\right) s+\left[\begin{array}{l}
\mathbf{n}_{p} \\
\mathbf{n}_{v}
\end{array}\right],
$$

resulting in a $4 L \times 1$ dimensional data model.

\section{Bartlett estimator}

The classical Bartlett estimator is possibly the most widely used technique for parameter estimation in signal processing, usually expressed in terms of the acoustic pressure. ${ }^{21}$

The Bartlett parameter estimate $\hat{\Theta}_{0}$ is given as the argument of the maximum of the function:

$$
P_{B, p}(\Theta)=E\left\{\hat{\mathbf{e}}_{p}^{H}(\Theta) \mathbf{y}_{p}\left(\Theta_{0}\right) \mathbf{y}_{p}^{H}\left(\Theta_{0}\right) \hat{\mathbf{e}}_{p}(\Theta)\right\},
$$

where $\mathbf{y}_{p}\left(\Theta_{0}\right)$ is the measured acoustic pressure data and the replica vector estimator $\hat{\mathbf{e}}_{p}\left(\Theta_{0}\right)$ defined as the vector $\mathbf{e}_{p}(\Theta)$ that maximizes the mean quadratic power:

$$
\hat{\mathbf{e}}_{p}(\Theta)=\arg \max _{\mathbf{e}_{p}(\Theta), \Theta \epsilon \Omega} \mathbf{e}_{p}^{H}(\Theta) \mathbf{R}_{p}\left(\Theta_{0}\right) \mathbf{e}_{p}(\Theta),
$$

subject to $\mathbf{e}_{p}^{H}(\Theta) \mathbf{e}_{p}(\Theta)=1$, where $(.)^{H}$ represents the complex conjugated transposed operator, $\Omega$ is the parameter space, $E\{$.$\} denotes statistical expectation and \mathbf{R}_{p}\left(\Theta_{0}\right)$ $=E\left\{\mathbf{y}_{p}\left(\Theta_{0}\right) \mathbf{y}_{p}^{H}\left(\Theta_{0}\right)\right\}$ is the correlation matrix.

In practice the correlation matrix $\mathbf{R}_{p}\left(\Theta_{0}\right)$ is usually unknown, thus a correlation matrix estimator $\hat{\mathbf{R}}_{p}\left(\Theta_{0}\right)$ is obtained by:

$$
\hat{\mathbf{R}}_{p}\left(\Theta_{0}\right)=\frac{1}{K} \sum_{k=1}^{K} \mathbf{y}_{p, k}\left(\Theta_{0}\right) \mathbf{y}_{p, k}^{H}\left(\Theta_{0}\right),
$$

assuming that there are $K$ data snapshots available.

Considering the acoustic pressure data model Eq. (14), the correlation matrix $\mathbf{R}_{p}\left(\Theta_{0}\right)$ can be written as:

$$
\mathbf{R}_{p}\left(\Theta_{0}\right)=\mathbf{h}_{p}\left(\Theta_{0}\right) \mathbf{h}_{p}^{H}\left(\Theta_{0}\right) \sigma_{s}^{2}+\sigma_{n}^{2} \mathbf{I} .
$$

Therefore, a possible estimator $\hat{\mathbf{e}}_{p}(\Theta)$ of $\mathbf{e}_{p}(\Theta)$ is obtained as:

$$
\hat{\mathbf{e}}_{p}(\Theta)=\arg \max _{\mathbf{e}_{p}(\Theta), \Theta \in \Omega}\left\{\mathbf{e}_{p}^{H}(\Theta) \mathbf{R}_{p}\left(\Theta_{0}\right) \mathbf{e}_{p}(\Theta)\right\},
$$

subject to $\mathbf{e}_{p}{ }^{H}(\Theta) \mathbf{e}_{p}(\Theta)=1$. According to (21) it can be shown that the well-known nontrivial solution ${ }^{22,23}$ is

$$
\hat{\mathbf{e}}_{p}(\Theta)=\frac{\mathbf{h}_{p}(\Theta)}{\sqrt{\mathbf{h}_{p}^{H}(\Theta) \mathbf{h}_{p}(\Theta)}},
$$

where the denominator is a scalar normalization and $\mathbf{h}_{p}(\Theta)$ contains the replica of the signal structure as "seen" by the receiver.

Replacing (23) and (21) in the generic estimator (18) provides the Bartlett estimator for acoustic pressure ( $p$-only) of any search parameter $\Theta$ :

$$
\begin{aligned}
P_{B, p}(\Theta) & =\frac{\mathbf{h}_{p}^{H}(\Theta) \mathbf{R}_{p}\left(\Theta_{0}\right) \mathbf{h}_{p}(\Theta)}{\mathbf{h}_{p}^{H}(\Theta) \mathbf{h}_{p}(\Theta)} \\
& =\frac{\mathbf{h}_{p}^{H}(\Theta) \mathbf{h}_{p}\left(\Theta_{0}\right) \mathbf{h}_{p}^{H}\left(\Theta_{0}\right) \mathbf{h}_{p}(\Theta)}{\mathbf{h}_{p}^{H}(\Theta) \mathbf{h}_{p}(\Theta)} \sigma_{s}^{2}+\sigma_{n}^{2} \\
& =B_{p}(\Theta) \sigma_{s}^{2}+\sigma_{n}^{2},
\end{aligned}
$$

where $B_{p}(\Theta)$ is the noise-free pressure beampattern (0 $\left.\leq B_{p}(\Theta) \leq 1\right)$, with the parameter estimator given by:

$$
\hat{\Theta}=\arg \max _{\Theta \in \Omega} P_{B, p}(\Theta) .
$$

The derivation of the Bartlett estimator for particle velocity only ( $v$-only) and for full VSA $(p+v)$ can be done by taking into account the data model (16), (17) and the maximization of the replica vector presented in the Appendix. Thus, the Bartlett estimator for $v$-only outputs can be written as:

$$
\begin{aligned}
P_{B, v}(\Theta) & =\frac{\left[\mathbf{u}^{H}(\Theta) \mathbf{u}\left(\Theta_{0}\right)\right]^{2}}{\mathbf{u}^{H}(\Theta) \mathbf{u}(\Theta)} B_{p}(\Theta) \sigma_{s}^{2}+\sigma_{n}^{2} \\
& \propto\left[\cos ^{2}(\delta)\right] P_{B, p}(\Theta),
\end{aligned}
$$

where $B_{p}(\Theta)$ is the beampattern for $p$-only defined in (24), $\delta$ is the angle between the replica vector $\mathbf{u}(\Theta)$ and the data vector $\mathbf{u}\left(\Theta_{0}\right)$, considering that the inner product between two vectors is proportional to the cosine of the angle between these vectors. Based on (26), one can conclude that the $v$-only Bartlett estimator response is proportional to the $p$-only Bartlett response, where the inner product $\mathbf{u}^{H}(\Theta) \mathbf{u}\left(\Theta_{0}\right)$ is the constant of proportionality herein called directivity factor. This directivity factor provides an improved sidelobe reduction or sidelobe suppression when compared with the $p$-only Bartlett response and contributes to improving the resolution of the parameter estimation.

The effect of merging the acoustic pressure and the particle velocity components in the previously derived Bartlett estimator gives the VSA $(p+v)$ Bartlett estimator, defined as

$$
\begin{aligned}
P_{B, p v}(\Theta)= & \frac{\left(\left[\begin{array}{c}
1 \\
\mathbf{u}(\Theta)
\end{array}\right]^{H}\left[\begin{array}{c}
1 \\
\mathbf{u}\left(\Theta_{0}\right)
\end{array}\right]\right)^{2}}{\left[\begin{array}{c}
1 \\
\mathbf{u}(\Theta)
\end{array}\right]^{H}\left[\begin{array}{c}
1 \\
\mathbf{u}(\Theta)
\end{array}\right]} B_{p}(\Theta) \sigma_{s}^{2}+\sigma_{n}^{2} \propto[1 \\
& +\cos (\delta)]^{2} P_{B, p}(\Theta) \propto\left[4 \cos ^{4}\left(\frac{\delta}{2}\right)\right] P_{B, p}(\Theta) .
\end{aligned}
$$

One can conclude that when the VSA $(p+v)$ estimator is considered, the estimate function is proportional to $\left[4 \cos ^{4}(\delta / 2)\right]$ providing a wider main lobe, shown in (27), as compared to the estimator with $v$-only (26). This is due to the cosine of the half angle. Moreover, the inclusion of the pressure on the estimator eliminates the ambiguities caused by the $\left[\cos ^{2}(\delta)\right]$ even when frequencies higher than the array design frequency are used. This behavior is illustrated with simulations in the next section. 


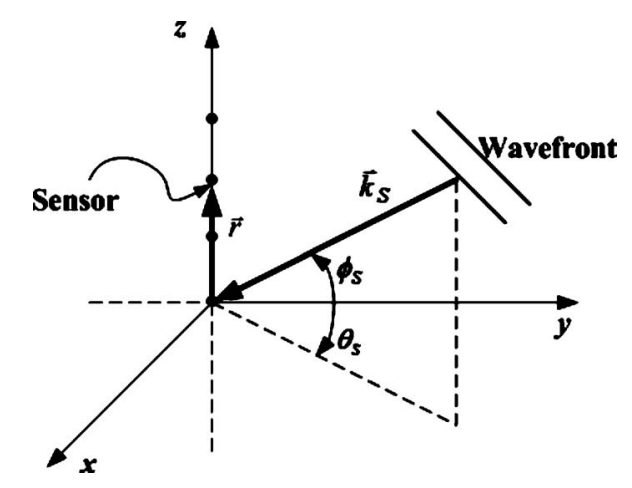

FIG. 2. Array coordinates and geometry of acoustic plane wave propagation, with azimuth $\left(\theta_{S}\right)$ and elevation $\left(\phi_{S}\right)$ angles. The vector sensor elements are located in the $z$-axis with the first element at the origin of the cartesian coordinate system.

\section{SIMULATION RESULTS}

In order to illustrate the advantage of vector sensors in parameter estimation using the VSA Bartlett estimator, two applications are discussed: direction of arrival (DOA) estimation and matched-field inversion (MFI) for ocean bottom properties estimation. The TRACE Gaussian beam model ${ }^{18}$ is used to generate the replica field. This model was designed to perform two dimensional acoustic ray tracing in ocean waveguides and provides different sets of output information such as the acoustic pressure and the particle velocity components.

\section{A. DOA estimation}

The main advantage of the VSA in DOA estimation is that it resolves both vertical and azimuthal directions with a linear array. The plane wave beamformer is applied to compare the performance of the VSA with that of pressure sensor arrays, where the individual sensor outputs are delayed, weighted and summed in a conventional manner. In the case of plane wave DOA estimation, the search parameter $\Theta$ is the direction $\left(\theta_{S}, \phi_{S}\right)$, where $\theta_{S}$ is the azimuth angle and $\phi_{S}$ is the elevation angle. The replica vectors in (24), (26), and (27) are simple combinations of weights, which are direction cosines for the particle velocity components and unity for pressure. These are, respectively given by:

- considering pressure only:

$$
\mathbf{e}_{p}\left(\theta_{S}, \phi_{S}\right)=\exp \left(i \overrightarrow{k_{S}} \cdot \vec{r}\right),
$$

- considering particle velocity components only:

$$
\mathbf{e}_{v}\left(\theta_{S}, \phi_{S}\right)=\left[\mathbf{u}\left(\theta_{S}, \phi_{S}\right)\right]^{T} \otimes \exp \left(i \overrightarrow{k_{S}} \cdot \vec{r}\right),
$$

where the weigthing vector is $\mathbf{u}\left(\theta_{S}, \phi_{S}\right)$ $=\left[\begin{array}{lll}\cos \left(\theta_{S}\right) \sin \left(\phi_{S}\right) \sin \left(\theta_{S}\right) \sin \left(\phi_{S}\right) \cos \left(\phi_{S}\right)\end{array}\right]^{T}$,

- and for both pressure and particle velocity:

$$
\mathbf{e}_{p v}\left(\theta_{S}, \phi_{S}\right)=\left[\begin{array}{ll}
1 & \mathbf{u}\left(\theta_{S}, \phi_{S}\right)
\end{array}\right]^{T} \otimes \exp \left(i \overrightarrow{k_{S}} \cdot \vec{r}\right),
$$

where $\overrightarrow{k_{S}}$ is the wavenumber vector corresponding to the chosen steering angle, or look direction $\left(\theta_{S}, \phi_{S}\right)$ of the array, $\theta_{S} \in[-\pi ; \pi], \phi_{S} \in[-\pi / 2 ; \pi / 2]$ and $\vec{r}$ is the position vector of the VSA elements as shown in Fig. 2. (a)

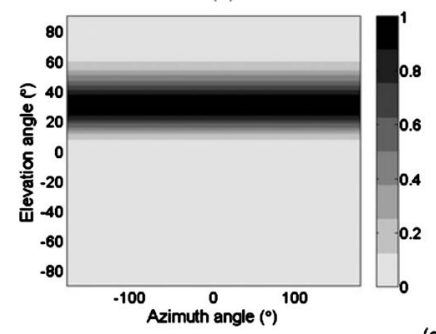

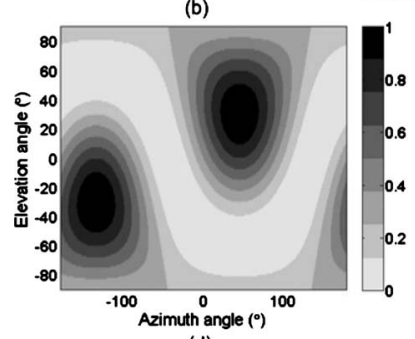

(d)

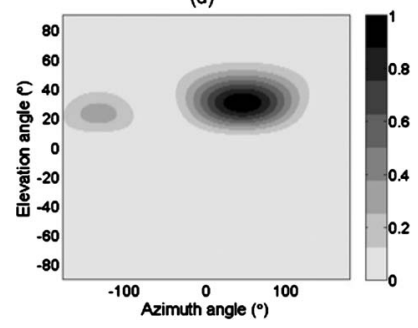

(c)

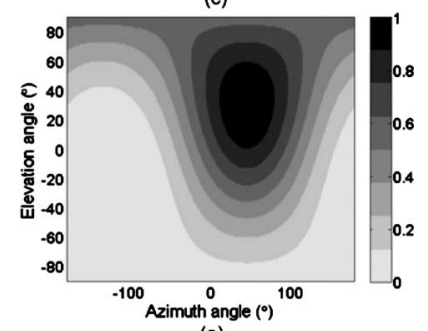

(e)

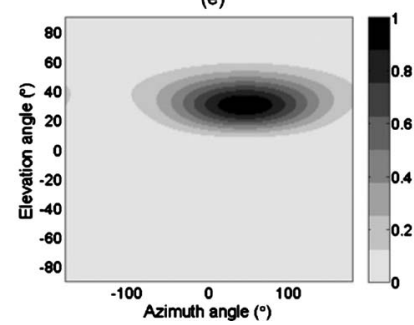

FIG. 3. DOA estimation simulation results at frequency $7500 \mathrm{~Hz}$ with azimuth $\left(\theta_{S}=45^{\circ}\right)$ and elevation $\left(\phi_{S}=30^{\circ}\right)$ angles using the normalized Bartlett beamformer considering: (a) $p$-only response, (b) the $\left[\cos ^{2}(\delta)\right]$ of Eq. (26), (c) the $\left[4 \cos ^{4}(\delta / 2)\right]$ of Eq. (27), (d) $v$-only (26), and (e) all sensors VSA $(p+v)(27)$.

The VSA has four equally spaced elements (with $10 \mathrm{~cm}$ spacing) and is located along the $z$-axis, with the first element at the origin of the cartesian coordinates system (Fig. 2 ). The simulation results were obtained for the array design frequency, $7500 \mathrm{~Hz}$, and for a true source DOA of $\theta_{S}=45^{\circ}$ and $\phi_{S}=30^{\circ}$. Figure 3 presents the simulation results when Eqs. (24), (26), and (27) are applied to DOA estimation.

Figure 3(a) illustrates the results when the $p$-only estimator is considered, using (24). Since the array is placed along the vertical axis, only the elevation angle is resolved due to the omnidirectionality of the pressure sensors. Figure $3(\mathrm{~b})$ and 3(c) present the results for the directivity factors $\left[\cos ^{2}(\delta)\right]$ and $\left[4 \cos ^{4}(\delta / 2)\right]$ in (26) and (27), respectively, where $\delta$ is the angle between the replica and the data vector (Section II C). It can be seen that the ambiguity presented in Fig. 3(b) is eliminated when $\left[4 \cos ^{4}(\delta / 2)\right]$ is used [Fig. 3(c)], due to the cosine of the half angle. Figure 3(d) shows the results of the Bartlett estimator using $v$-only - this yields the best resolution in both azimuth and elevation, but produces a low amplitude ambiguity at an azimuth of $\left(-135^{\circ}\right)$. On the other hand, the VSA $(p+v)$ Bartlett estimator has a wider main lobe without ambiguities, as shown in Fig. 3(e), and the DOA is resolved in both elevation and azimuth. The results presented in Fig. 3(d) and 3(e) are obtained by Eqs. (26) and (27), but they can be seen by visual superposition of Fig. 3(a) with Figs. 3(b) and 3(c), respectively. Both directions are resolved and the conjugation of the acoustic pressure with the particle velocity eliminates the ambiguity with an array of only a few elements.

Santos et al:: Seabed characterization with a vector sensor array 


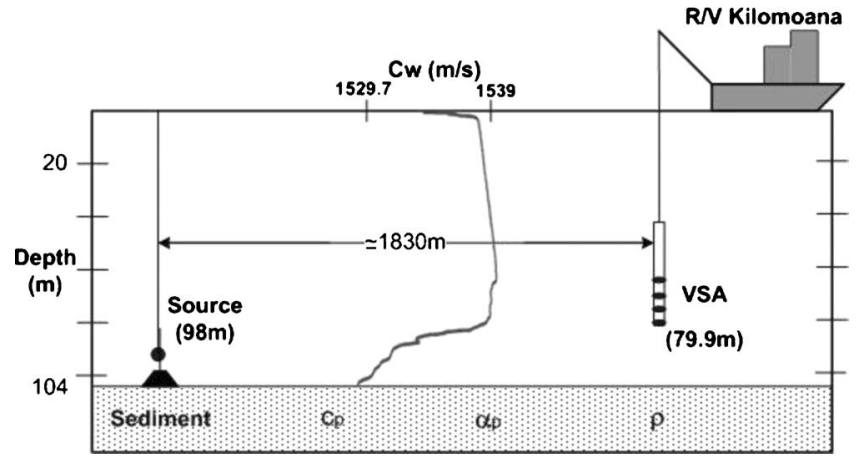

FIG. 4. Simulation scenario based on the typical setup encountered during the Makai experiment with a very large mixed layer, characteristic of Hawaii. The source is bottom moored at $98 \mathrm{~m}$ depth and $1830 \mathrm{~m}$ range. The VSA is deployed with the deepest element at $79.9 \mathrm{~m}$.

\section{B. Ocean bottom properties MFI}

The simulation scenario is shown in Fig. 4 and is partially based on the Makai experimental setup (for which results on experiment data will be presented in Section IV). This environment has a deep mixed layer, characteristic of Hawaii, and the bathymetry at the site is range independent with a water depth of $104 \mathrm{~m}$. The source is bottom moored at $98 \mathrm{~m}$ depth and $1830 \mathrm{~m}$ range. The results are obtained with a four element $(10 \mathrm{~cm}$ spacing) VSA deployed with the deepest element positioned at $79.9 \mathrm{~m}$. The frequency of $13078 \mathrm{~Hz}$ is considered in the simulations.

A common approach in acoustic inversion is to consider both geometrical and environmental parameters as candidates for inversion. However, during the Makai experiment the system geometry was known to a degree of accuracy that allowed to consider only environmental inversion while keeping fixed the geometrical parameters. Moreover, since the objective is seabed characterization and the Makai data set can be well described with a simplified three parameter seabed model, the seabed parameters to be considered herein are sediment compressional speed $\left(c_{p}\right)$, density $(\rho)$ and compressional attenuation $\left(\alpha_{p}\right)$. These parameters are estimated taking into account the particle velocity outputs and a MFI processor based on the Bartlett estimators presented in Section II C. The field replicas are generated using the TRACE Gaussian beam model ${ }^{18}$ for a half space seabed.

Preliminary tests have shown that the MFI processor is decreasingly sensitive to sediment compressional speed, density and nearly insensitive to compressional attenuation. Therefore the parameter space was reduced to the most sensitive parameters: sediment compressional speed and density. The true values for the seabed parameters considered in the simulation were taken as: $c_{p}=1575 \mathrm{~m} / \mathrm{s}, \rho=1.5 \mathrm{~g} / \mathrm{cm}^{3}$ and $\alpha_{p}=0.6 \mathrm{~dB} / \lambda$. To illustrate and to compare the resolution of the several seabed parameter estimators previously defined, Fig. 5 presents the ambiguity surfaces 1D cross sections for each parameter.

Figure 5(a) and 5(b) compare the estimation performance obtained considering the $p$-only [Eq. (24)] for 4 and 16 pressure sensors and the VSA $(p+v)$ [Eq. (27)], for sedi- (a)

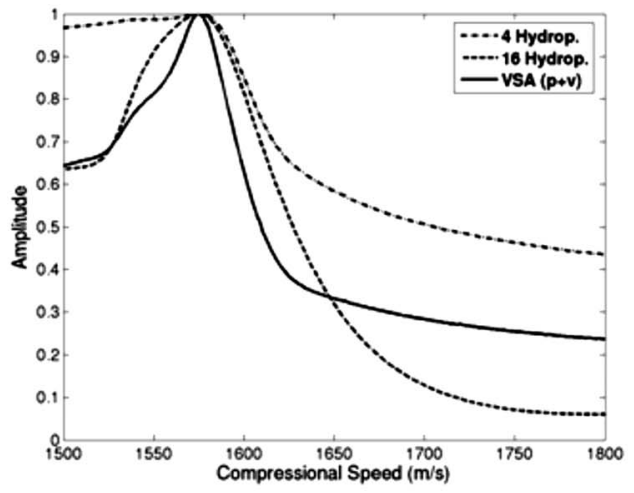

(c)

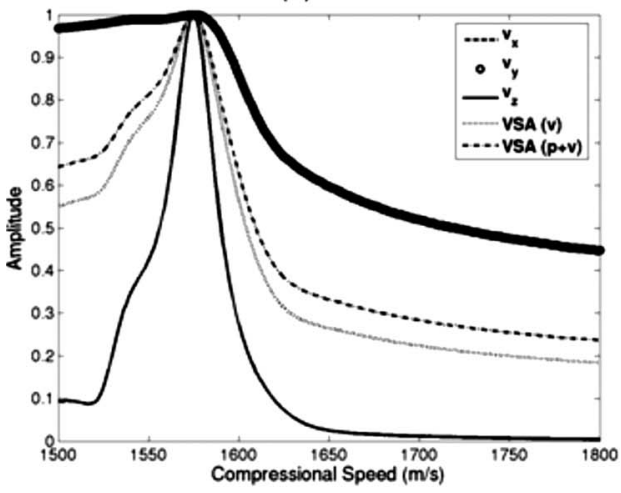

(b)

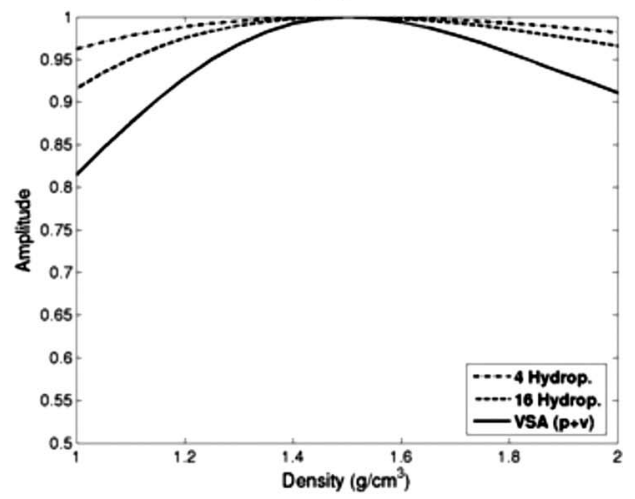

(d)

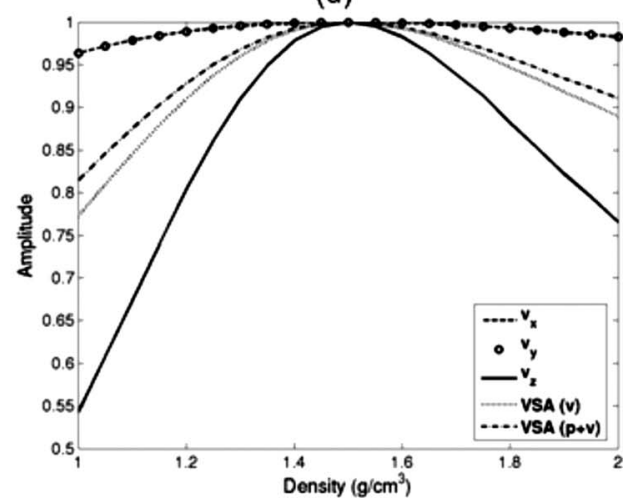

FIG. 5. Seabed parameters estimation simulation results obtained with the normalized Bartlett estimator at frequency $13078 \mathrm{~Hz}$ and for $\alpha=0.6 \mathrm{~dB} / \lambda$ for $[(\mathrm{a})$ and (c)] sediment compressional speed where $\rho=1.5 \mathrm{~g} / \mathrm{cm}^{3}$ and $\left[(\mathrm{b})\right.$ and (d)] density where $c_{p}=1575 \mathrm{~m} / \mathrm{s}$. The simulation results were obtained comparing: the $p$-only Bartlett estimator response (24) for 4 and 16 pressure sensors with VSA $(p+v)$ Bartlett estimator response (27) (a) and (b) and the Bartlett estimator response considering: individual data components $\left(v_{x}, v_{y}\right.$ and $\left.v_{z}\right), v$-only Bartlett estimator (VSA $\left.(v)\right)$ and all sensors (VSA $\left.(p+v)\right)(\mathrm{c})$ and (d). 


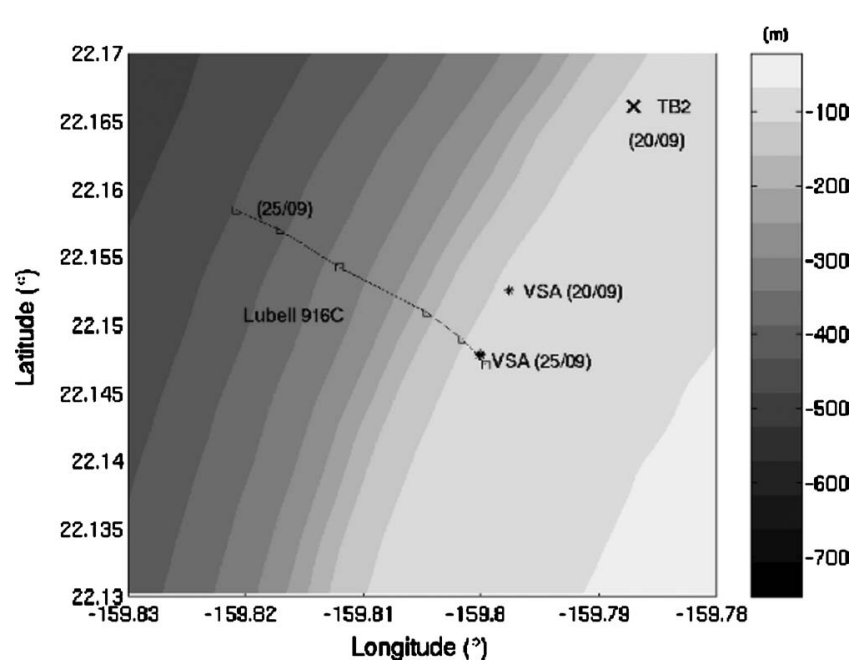

FIG. 6. Bathymetry map of the Makai Experiment area with the location of the VSA for the two deployments (September 20th and 25th) as well as the location of the acoustic sources TB2 (fixed) and Lubel1916C (track).

ment compressional speed and density, respectively. The scope of comparing the 4-element VSA with a 4 and 16 pressure sensor array is to consider an equal aperture comparison in the former and an equal number of sensors in the later. The figures show that the VSA improves the resolution in both sediment compressional speed and density, when compared with 4 and 16 pressure sensors. The results suggest that the VSA can offer a significant array size reduction with a better performance when compared with a pressure sensor array.

Figure 5(c) and 5(d) show the estimation results, obtained respectively, for sediment compressional speed and density, considering the Bartlett estimator for: individual particle velocity component, $v$-only and VSA $(p+v)$. The plots obtained for horizontal particle velocity components $v_{x}$ (dashed line) and $v_{y}$ (circles) are coincident, since these components mostly depend on low-order modes, thus having little or no interaction with the bottom. On the other hand, the vertical component $v_{z}$ (solid line) has a higher sensitivity to bottom structure than the $v_{x}$ and $v_{y}$ components, since it is influenced by the high-order modes with a larger contribution to the vertical particle velocity due to their grazing angle. ${ }^{11}$ The results suggest that both seabed parameters can be obtained using only the vertical component of the particle velocity. Comparing the VSA Bartlett outputs VSA $(v)$ and VSA $(p+v)$, the former [dotted curve in Fig. 5(c) and 5(d)] has a narrower main lobe than the later (dashdot curve) due to the cosine function of the half angle, [Eq. (27)], similarly to the result obtained in DOA estimation. One can conclude that a VSA increases significantly the resolution of the seabed parameters: sediment compressional speed and density.

\section{EXPERIMENTAL RESULTS}

\section{A. Experimental setup}

The data analyzed here were acquired during the Makai Experiment that took place from September 15th to October 2nd 2005, on the North West coast of Kauai I., Hawaii, USA. ${ }^{15}$ Figure 6 shows the bathymetry map of the MakaiEx

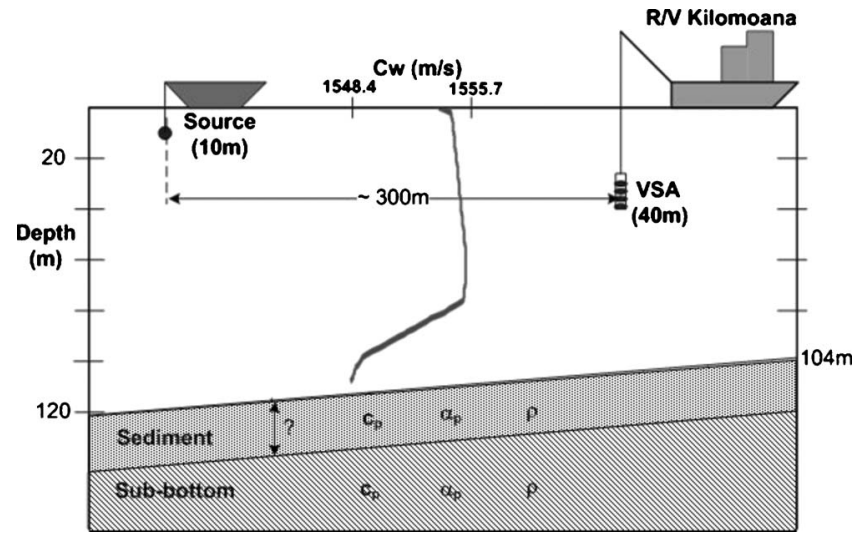

FIG. 7. Baseline environment on September 25th 2005 with mean sound speed profile. The VSA was deployed with the deepest element at $40 \mathrm{~m}$ and the Lubel1916C source was towed from a boat at $10 \mathrm{~m}$ depth.

area, showing a smooth and uniform area of depth around 80-100 m accompanying the island bathymetric contour, surrounded by the continental relatively steep slope to the deeper ocean to the West. Extensive ground truth measurements were carried out in this area during previous experiments and showed that most of the bottom in the area is covered with coral sands over a basalt hard bottom. The sound velocity in coral sands should be approximately 1700 $\mathrm{m} / \mathrm{s}$ and the sediment thickness is unknown but expected to be on the order of a fraction of a meter in most places.

The four-element $10 \mathrm{~cm}$ spacing vertical VSA was deployed twice, on September 20th and 25th and acquired data from two different acoustic sources: the TB2 testbed and the Lubell916C, respectively. Figure 6 also shows the equipment location: on September 20th, the VSA and TB2 were in a fixed-fixed configuration over a range independent bathymetry with water depth of approximately 104 m; on September 25th, the VSA was fixed and the Lubell 916C was towed toward the VSA by a small inflatable boat at a depth of $10 \mathrm{~m}$ starting at a range of $2300 \mathrm{~m}$ (track marked Lubel1916C).

On September 20th, the experimental baseline environment with the mean sound speed profile considered for this day is identical to that shown in Fig. 4. The VSA was deployed with the deepest element at $79.9 \mathrm{~m}$ depth and the source TB2 was bottom moored at $98 \mathrm{~m}$ depth and $1830 \mathrm{~m}$ range. The experimental setup used in September 25th, is shown in Fig. 7, where the VSA was deployed with the deepest element at $40 \mathrm{~m}$ depth. The Lubell 916C source approached the VSA from $2300 \mathrm{~m}$ range but the data considered herein was taken at $300 \mathrm{~m}$ range, where the water depth varies from $119 \mathrm{~m}$ at the source location to $104 \mathrm{~m}$ at the VSA. On September 20th, the TB2 source transmitted linear frequency modulation sweeps (LFMs), tone combs and M-sequences in the $8-14 \mathrm{kHz}$ band, while only the tone combs were used in the processing for this day. On September 25th, the Lubell 916C source transmitted sequences of controlled waveforms - of these, the $50 \mathrm{~ms}$ LFM pulses spanning the $8-14 \mathrm{kHz}$ band were processed.

\section{B. Seabed parameter estimation}

The estimation of seabed parameters is performed using two different methods. First, taking advantage of the spatial 


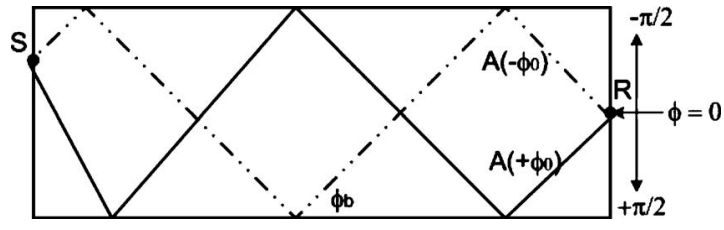

FIG. 8. Sketch of the ray approached geometry of a plane wave emitted by source $(\mathrm{S})$ and received by the receiver $(\mathrm{R})$ at the elevation angle $\phi_{0}$.

filtering capabilities and directionality of the VSA, the seabed parameters and layer structure is estimated comparing the reflection loss of experimental data to the reflection loss modeled by SAFARI model. ${ }^{24}$ Second, the seabed parameters are estimated using MFI comparing several Bartlett estimators previously derived.

\section{Bottom reflection loss}

C. Harrison ${ }^{25}$ proposed an estimation technique for bottom reflection loss, using vertical pressure sensor array measurements of surface generated noise. The estimation of bottom reflection loss versus grazing angle for the signal bandwidth is obtained by dividing the down and upward energy reaching the array. This technique can be adapted for vertical measurements of an azimuthal direction of acoustic sources with a small aperture VSA, taking advantage of its spatial filtering capabilities. The bottom reflection loss deduced from experimental data is then compared to the reflection loss modeled by the SAFARI model, for a given set of parameters for sediment and/or bottom: compressional wave speed $c_{p}$, shear wave speed $c_{S}$, compressional wave attenuation $\alpha_{p}$, shear attenuation $\alpha_{S}$ and density $\rho$. The best agreement gives an estimate of bottom layering structure together with its most characteristic physical parameters.

Let us consider an emitted signal $S$ in a range independent environment, where the localization is a function of elevation angle $\phi$ and azimuth $\theta$. The array beam pattern $B(\theta, \phi)$ for the source look direction was estimated taking into account the plane wave beamforming described in Section III A. Then, the array beam pattern for an azimuthal direction $\theta$, shown in Fig. 8 , is given by the vertical beam response $A\left(\phi_{0}\right)$ for each steer angle $\phi_{0}$. According to ${ }^{25}$ the ratio between the downward and upward beam responses is an approximation of the bottom reflection coefficient $R_{b}$ :

$$
\frac{A\left(-\phi_{0}\right)}{A\left(+\phi_{0}\right)}=R_{b}\left(\phi_{b}\left(\phi_{0}\right)\right),
$$

where the angle measured by beamforming at the receiver $\phi_{0}$, is corrected to the angle at the seabed $\phi_{b}$, in agreement to the sound-speed profile by Snell's law.

$$
\phi_{b}=\arccos \left[\left(\frac{c_{b}}{c_{r}}\right) \cos \left(\phi_{0}\right)\right],
$$

where $c_{b}$ and $c_{r}$ are the sound speed at the bottom and at the receiver, respectively.

Figure 9 presents the vertical beam response for each frequency extracted for the source azimuthal direction of interest, considering both $p$-only Fig. 9(a) and VSA $(p+v)$ Fig. 9(b). As it can be seen in Fig. 9(a), the beam response in the
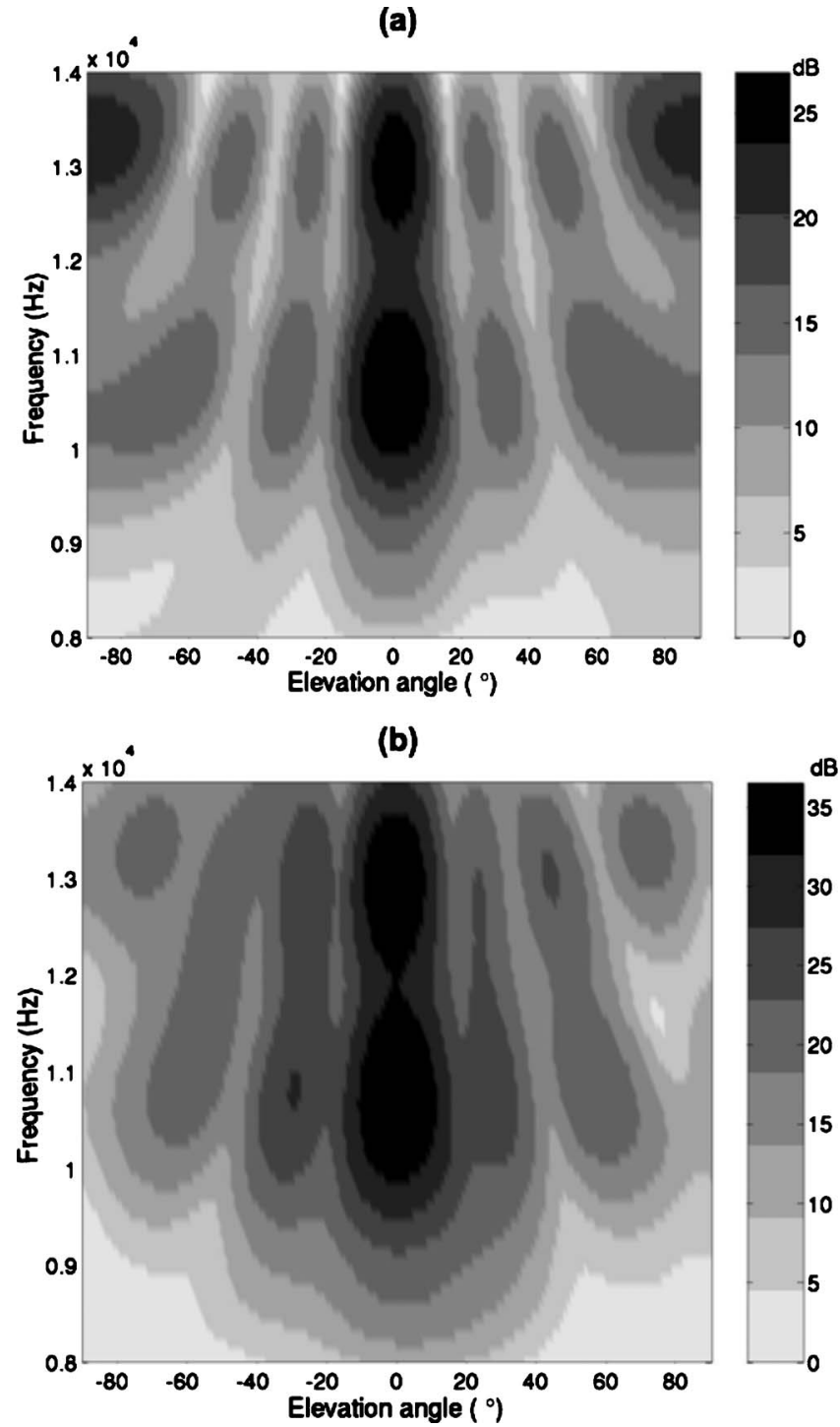

FIG. 9. Beam response at source azimuthal direction obtained using (a) the $4 p$-only sensors of the VSA and (b) all sensors VSA $(p+v)$.

$p$-only case is nearly symmetric for both negative and positive elevation angles (up and down, respectively), resulting in a poor information about the bottom attenuation. Comparing with the directional case, Fig. 9(b), the vertical beam response clearly differentiates up and downward energy. This allows for retrieving bottom information, since the vertical component of the VSA is constituted by the rays with strong interactions with the seabed. This is clearly a unique capability resulting from the processing gain provided by vector sensors.

Dividing the down to up beam responses for the same elevation angle, the frequency versus bottom angle reflection losses curves were obtained for the signals acquired on September 25th and then compared with the reflection loss curves modeled by the SAFARI model, ${ }^{24}$ using a trial and error approach. Initial values of the parameters were found in the literature based on available qualitative description of the area. ${ }^{19}$ Then, manual adjustments were made to estimate a reflection loss figure similar to that obtained with measured data. It was observed that the most relevant parameters are 
(a)

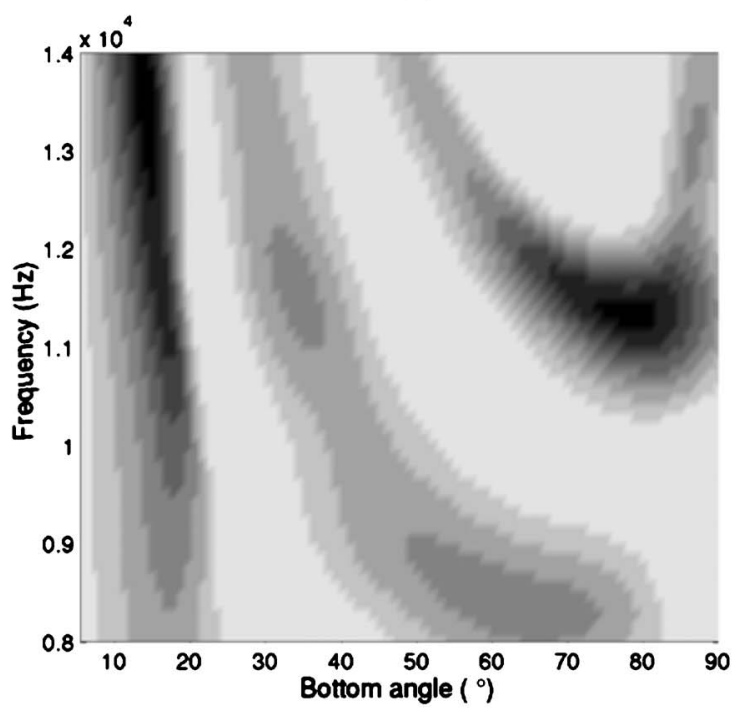

(b)

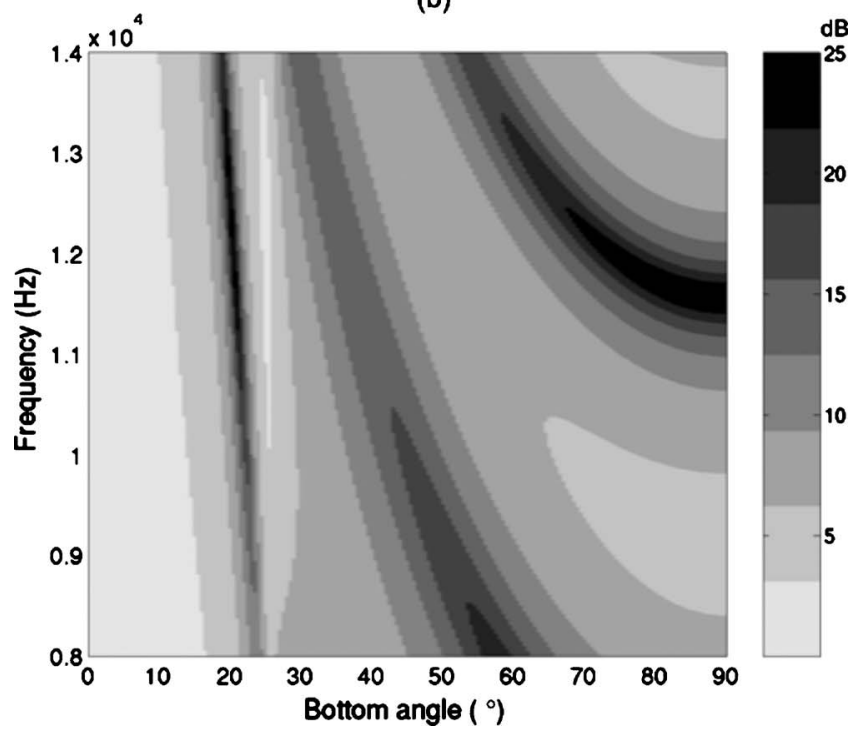

FIG. 10. Bottom reflection loss: deduced from the down-up ratio of the experimental data (a) and modeled by the SAFARI model (b).

the layer thickness, an important parameter for fringe separation agreement, and the sound speed on the various layers and in the half-space, which influences the critical angles of the form:

$$
\phi_{c_{i}}=\arccos \left(\frac{c_{W}}{c_{s_{i}}}\right),
$$

where $c_{W}$ is the water sound speed near the water sediment interface and $c_{s_{i}}$ is the $i$ th sediment or sub-bottom sound speed.

Figure 10(a) and 10(b) presents the bottom reflection loss deduced from the down-up ratio of the experimental data and that modeled by the SAFARI model, respectively. This structure suggests that the area can be modeled as a four-layer environment (three boundaries): water, two sediments and the bottom half space. Three critical angles are presented in Fig. 10(a): $\phi_{c_{1}} \simeq 13^{\circ}, \phi_{c_{2}} \simeq 26^{\circ}$ and $\phi_{c_{3}}$ $\simeq 49^{\circ}$ and according to (33) where the water sound speed is
TABLE I. Estimatted bottom parameters taking into account the measured VSA data on September 25th and manual adjustments on SAFARI model, considering four layer structure.

\begin{tabular}{lccc}
\hline \hline Sediment & First layer & Second layer & Sub-bottom \\
\hline Thickness $(\mathrm{m})$ & 0.175 & 20 & $\ldots$ \\
$\rho\left(\mathrm{g} / \mathrm{cm}^{3}\right)$ & 1.6 & 2.1 & 2.1 \\
$c_{p}(\mathrm{~m} / \mathrm{s})$ & 1570 & 1700 & 2330 \\
$c_{S}(\mathrm{~m} / \mathrm{s})$ & 67 & 700 & 1000 \\
$\alpha_{p}(\mathrm{~dB} / \lambda)$ & 0.6 & 0.1 & 0.1 \\
$\alpha_{S}(\mathrm{~dB} / \lambda)$ & 1.0 & 0.2 & 0.2 \\
\hline \hline
\end{tabular}

$c_{W}=1530 \mathrm{~m} / \mathrm{s}$, the sediment and sub-bottom sound speeds can be calculated: $c_{s_{1}}=1570 \mathrm{~m} / \mathrm{s}, c_{s_{2}}=1700 \mathrm{~m} / \mathrm{s}$ and $c_{s_{3}}$ $=2330 \mathrm{~m} / \mathrm{s}$. Figure 10(b) presents the reflection loss modeled with the same features as those observed in the experimental data, Fig. 10(a). Table I presents the results of the estimated bottom structure taking into account the real data and manual adjustments on the SAFARI model. The same fringe separation appears for a layer thickness of $0.175 \mathrm{~m}$ and this is in line with the ground truth (see Section IV A) but the first sediment sound speed is different from $1700 \mathrm{~m} / \mathrm{s}$, however, the sediment sound speed of $c_{s}=1570 \mathrm{~m} / \mathrm{s}$ is in line with the results obtained by MFI in the next section. One can conclude that the three-layer environment suggested in ${ }^{15}$ could be in fact a four-layer environment (water, soft sediment, sand and basalt) with a soft sediment over the sand. Due to the small value of the thickness of this first sediment, it was not considered in the descriptive ground truth measurements (see Section IV A).

\section{MFI results}

The vector sensor based MFI method discussed in Sections II C and III is applied to the data measured by the VSA on September 20th. In the simulation section it was found that compressional attenuation is the parameter with the least sensitivity and therefore the most difficult to invert for. Extensive runs were made for determining the most likely value for compressional attenuation within the range of 0.1 to $0.9 \mathrm{~dB} / \lambda$ and a best match was found for the value of $0.6 \mathrm{~dB} / \lambda$, which was then used as a fixed parameter in the sequel.

Figure 11 shows the ambiguity surfaces cross sections for sediment compressional speed obtained for the maximum density values of the estimator functions throughout almost two hours. Figure 11(a)-11(c) were obtained with the Bartlett estimators: [Eq. (26)] with the vertical component only, $v$-only [Eq. (26)] and VSA $(p+v)$ [Eq. (27)], respectively. These plots show the stability of the results during the data acquisition period (approximately $2 \mathrm{~h}$ ) and give an estimation of sediment compressional speed of approximately 1575 $\mathrm{m} / \mathrm{s}$. As already seen in the simulations, the vertical component has also a narrow main lobe due to the higher sensitivity to bottom structure, Fig. 11(a). On the other hand, the VSA $(p+v)$ estimator, Fig. 11(c), has a wider main lobe than the $v$-only estimator, Fig. 11(b), confirming the simulations and the analytical results obtained. The results are generically in good agreement with those obtained in the previous section. 
(a)

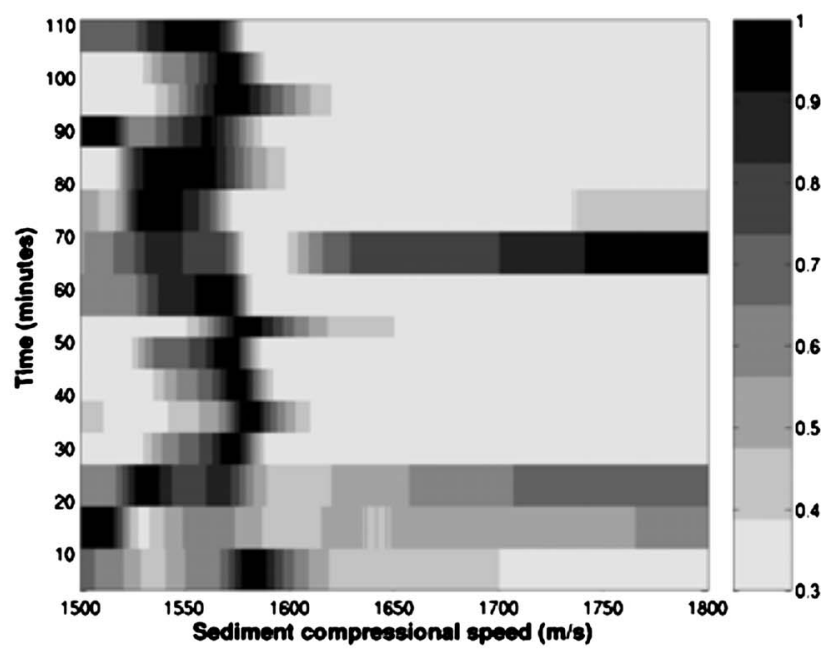

(b)

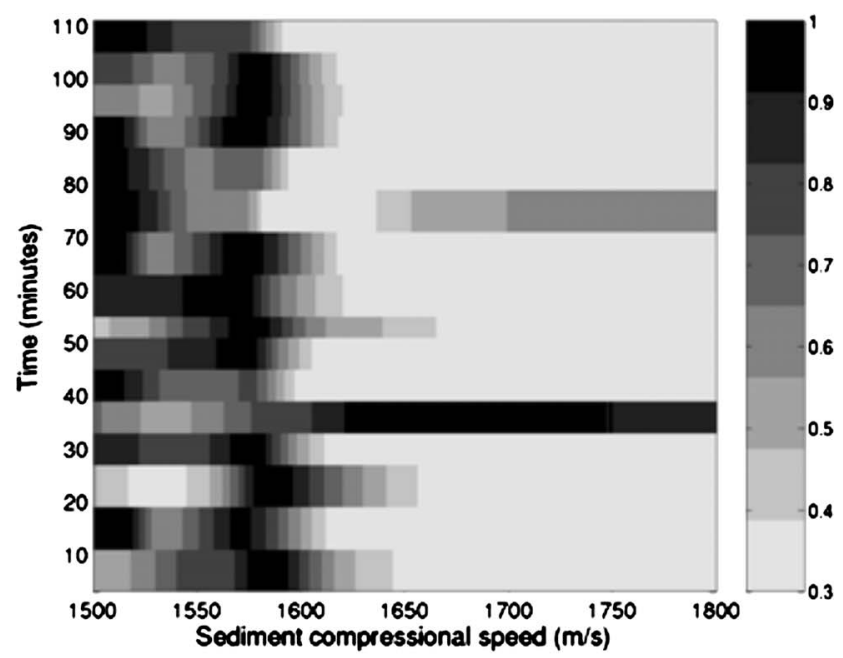

(c)

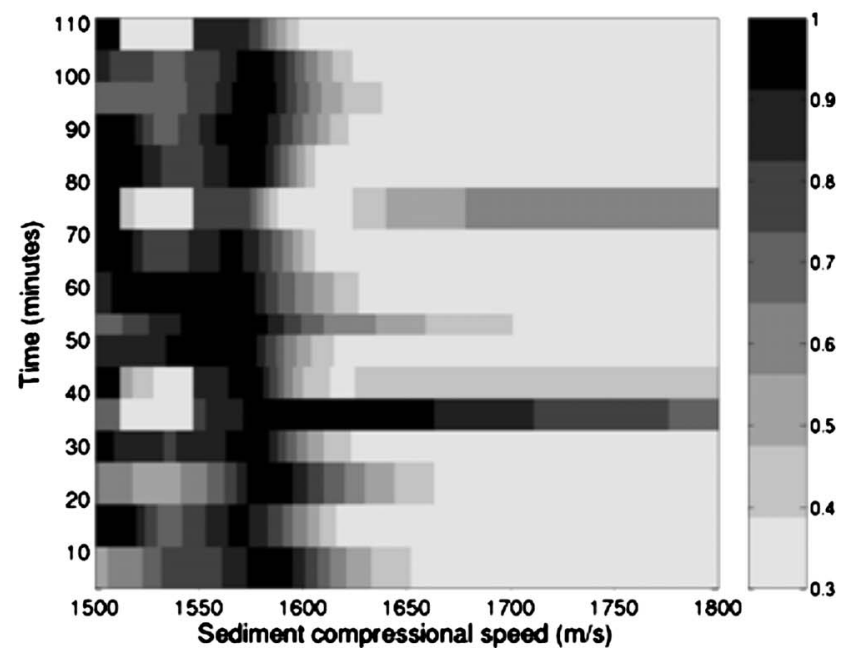

FIG. 11. Measured data normalized ambiguity surfaces for sediment compressional speed during data acquisition period, for: (a) Bartlett vertical component, (b) Bartlett VSA $(v)$ and (c) Bartlett VSA $(p+v)$.

Figure 12 shows the ambiguity surface for both seabed parameters and was obtained for the same estimator cases as in Fig. 11, using the geometric mean of the estimates along (a)

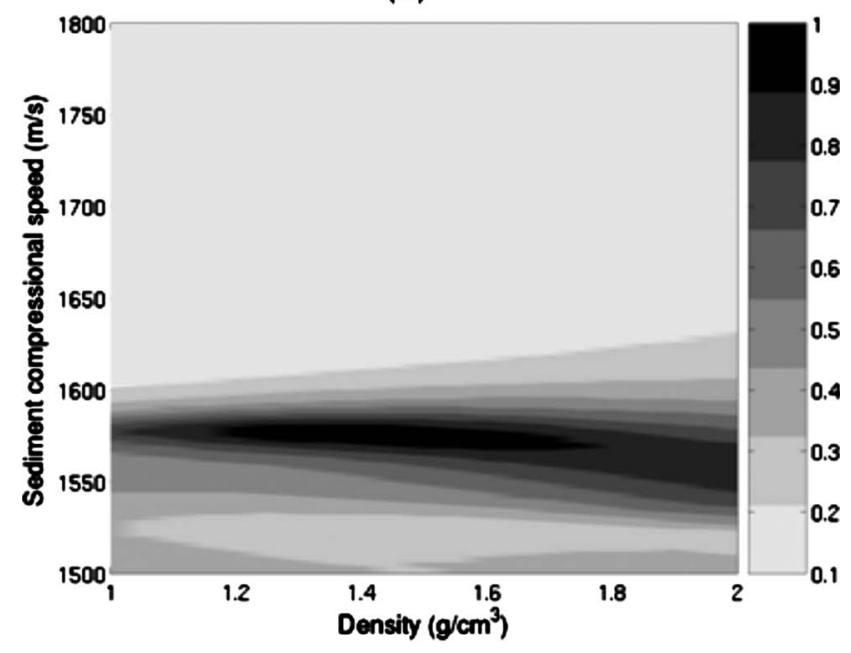

(b)

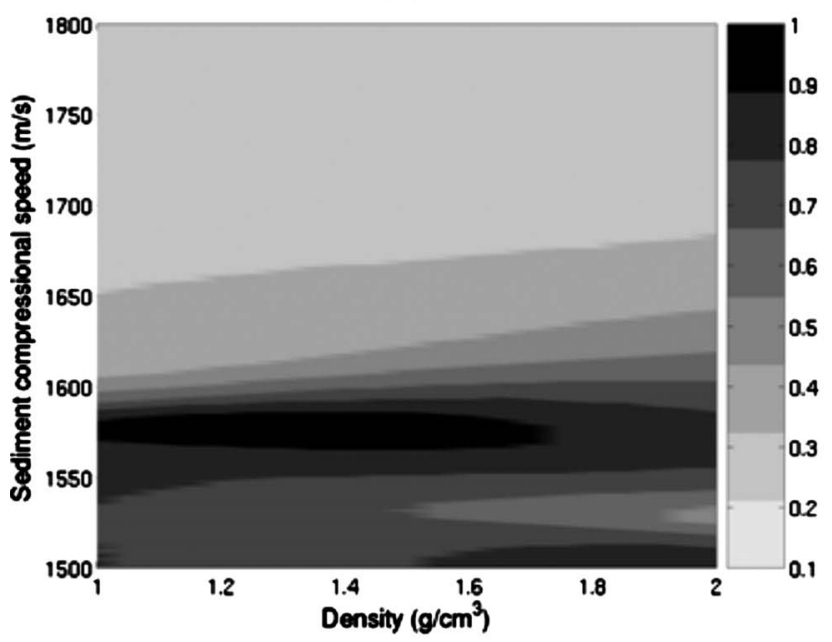

(c)

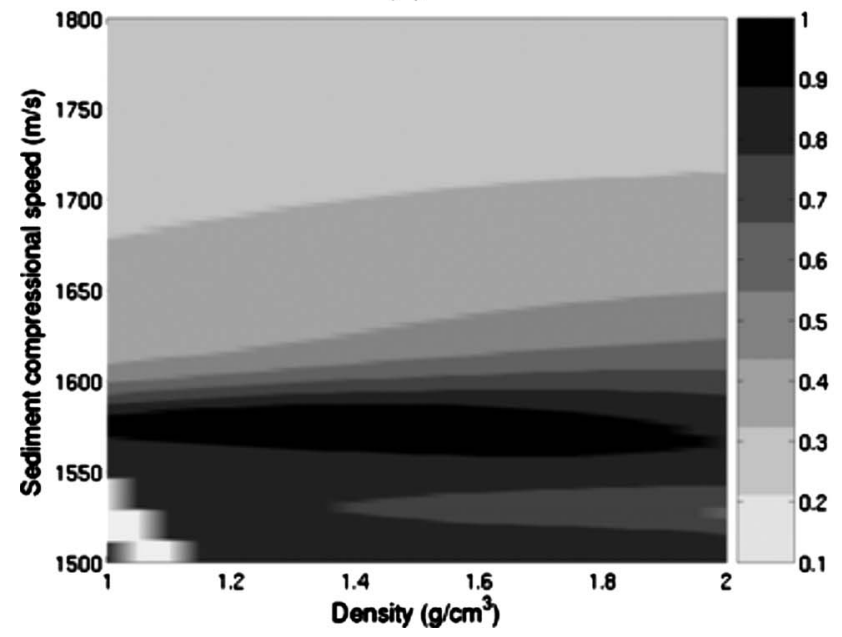

FIG. 12. Measured data normalized ambiguity surfaces for sediment compressional speed and density, using the geometric mean of estimates along the acquisition period, for: (a) Bartlett vertical component only, (b) Bartlett $\operatorname{VSA}(v)$ and (c) Bartlett VSA $(p+v)$.

the data acquisition period. Figure 12(a) shows the ambiguity surface considering [Eq. (26)] with only the vertical component of the particle velocity which points to values for den- 
sity of approximately $1.4 \mathrm{~g} / \mathrm{cm}^{3}$ and this component has a narrower main lobe. The VSA Bartlett estimators, [Eq. (26)] and [Eq. (27)], respectively Fig. 12(b) and 12(c) confirm this result but with wider main lobes. The results show that the density can be estimated with higher resolution using a small VSA than using a small pressure sensor array, and agree with the results obtained for bottom reflection loss (Section IV B 1).

\section{v. CONCLUSIONS}

In recent years, acoustic particle velocity sensors have been introduced and discussed by several authors, mainly in the DOA estimation context. In this paper, the possibility of using a vertical VSA and active signals in the $8-14 \mathrm{kHz}$ band to estimate bottom properties, beyond DOA estimation was presented. The original contributions of this work consist in the following: a vector sensor measurement model was developed, which allowed to introduce the particle velocity in the Bartlett estimator; it was demonstrated with two different estimation techniques, that the inclusion of the particle velocity information improves the resolution of the seabed parameter estimation; finally that good results can be obtained using only the vertical particle velocity component.

The determination of the bottom reflection coefficient deduced by the ratio of the downward and upward beam response $^{25}$ using $8-14 \mathrm{kHz}$ band measured data became a simple method for estimating the bottom structure and respective geoacoustic parameters. The unique capability of the VSA for vertical beam response information extraction, when compared with the performance of a pressure sensor array with the same aperture, was demonstrated. The reflection loss curves observed with the measured data were compared with the reflection loss curves predicted by the SAFARI model, which allowed to define the number of layers, their thickness and their geoacoustic parameters.

The proposed inversion based on VSA matched-field processing were used to estimate the values of the sediment parameters: sediment compressional speed, density and compressional attenuation. The classical Bartlett estimator adapted to vector sensor information provides better results for seabed parameter estimation than pressure sensor arrays of the same length. The estimates of sediment compressional speed produced from the vertical particle velocity component had high resolution and were consistent over a significant time interval. Furthermore, the VSA-based measurements also produced reliable estimates of sediment density and compressional attenuation. Note that these parameters are normally difficult to estimate with pressure measurements alone. An interesting and perhaps surprising outcome of this work was that the channel impulse response has sufficient structure to support estimation of seabed geoacoustic parameters in this high-frequency band. The results are compatible with those obtained with the bottom reflection curves and with the historical data of the area.

The particle velocity information enhances DOA and seabed geoacoustic parameter estimation, resulting in a better parameter resolution. The usage of VSA at high- frequency provides an alternative for a compact and easy-todeploy system in various underwater acoustical applications.

\section{ACKNOWLEDGMENTS}

The authors would like to thank Michael Porter, chief scientist for the Makai Experiment and Jerry Tarasek at Naval Surface Weapons Center for the loan of the vector sensor array used in the experiment. The authors also thank Bruce Abraham at Applied Physical Sciences for providing assistance with the data acquisition and the team at HLS Research, particularly Paul Hursky for their help with the data processed in this work. This work was partially supported by the project SENSOCEAN funded by FCT programme PTDC/EEA-ELC/104561/2008.

\section{APPENDIX: DERIVATION OF THE BARTLETT ESTIMATOR FOR PARTICLE VELOCITY}

For the derivation of the Bartlett estimator taking into account the particle velocity components, the following properties of the Kronecker product are considered:

1. $\mathbf{A} \otimes(a \mathbf{B})=(a \mathbf{A}) \otimes \mathbf{B}=a(\mathbf{A} \otimes \mathbf{B})$ where $a$ is a scalar,

2. $(\mathbf{A} \otimes \mathbf{B})^{H}=\mathbf{A}^{H} \otimes \mathbf{B}^{H}$,

3. $(\mathbf{A} \otimes \mathbf{B})(\mathbf{C} \otimes \mathbf{D})=\mathbf{A C} \otimes \mathbf{B D}$

4. if $\mathbf{A}_{1}, \mathbf{A}_{2}, \cdots, \mathbf{A}_{p}$ are $M \times M$ and $\mathbf{B}_{1}, \mathbf{B}_{2}, \cdots, \mathbf{B}_{p}$ are $N$ $\times N$ then $\left(\mathbf{A}_{1} \otimes \mathbf{B}_{1}\right)\left(\mathbf{A}_{2} \otimes \mathbf{B}_{2}\right) \cdots\left(\mathbf{A}_{p} \otimes \mathbf{B}_{p}\right)=\left(\mathbf{A}_{1} \mathbf{A}_{2} \cdots \mathbf{A}_{p}\right)$ $\otimes\left(\mathbf{B}_{1} \mathbf{B}_{2} \cdots \mathbf{B}_{p}\right)$.

In the following, $\mathbf{v}\left(\Theta_{0}, \Theta\right)=\mathbf{u}\left(\Theta_{0}, \Theta\right)$ when only particle velocity components are considered in the data model- $v$-only; or $\mathbf{v}\left(\Theta_{0}, \Theta\right)=\left[\begin{array}{ll}1 & \mathbf{u}\left(\Theta_{0}, \Theta\right)\end{array}\right]^{T}$ when both pressure and particle velocity components are consideredVSA $(p+v)$. For simplicity, the following notation $\mathbf{v}\left(\Theta_{0}\right)$ $\rightarrow \mathbf{v}_{\mathbf{0}}$ and $\mathbf{v}(\Theta) \rightarrow \mathbf{v}$ are used.

The correlation matrix $\mathbf{R}_{\mathbf{0}}$ depending on the particle velocity data model, with or without pressure, can be written as:

$$
\mathbf{R}_{\mathbf{0}}=\left[\mathbf{v}_{\mathbf{0}} \otimes \mathbf{h}_{\mathbf{0} p}\right]\left[\mathbf{v}_{\mathbf{0}} \otimes \mathbf{h}_{\mathbf{0} p}\right]^{H} \sigma_{s}^{2}+\sigma_{n}^{2} \mathbf{I},
$$

where the additive noise is zero mean, white both in time and space, with variance $\sigma_{n}^{2}$ and uncorrelated with the signal $s$, itself with zero mean and variance $\sigma_{s}^{2}, \mathbf{h}_{\mathbf{0} p}$ is the channel frequency response at the $L$ pressure sensors and $\mathbf{v}_{\mathbf{0}}$ is the data vector.

A possible estimator $\hat{\mathbf{e}}$ of $\mathbf{e}$ is obtained as:

$$
\hat{\mathbf{e}}=\arg \max _{\mathbf{e}}\left\{\mathbf{e}^{H} \mathbf{R}_{\mathbf{0}} \mathbf{e}\right\},
$$

subject to $\mathbf{e}^{H} \mathbf{e}=1$.

Using the eigen decomposition of the correlation matrix associated with the signal and noise subspaces according to structure (A1) and for this case in particular, it can be shown that $\mathbf{v}_{\mathbf{0}} \otimes \mathbf{h}_{\mathbf{0}}$ p is one of the eigenvectors of $\mathbf{R}_{\mathbf{0}}$, since postmultiplying (A1) by this eigenvector and using the properties of the Kronecker product 2 and 3, gives:

Santos et al:: Seabed characterization with a vector sensor array 


$$
\begin{aligned}
\mathbf{R}_{\mathbf{0}}\left[\mathbf{v}_{\mathbf{0}} \otimes \mathbf{h}_{\mathbf{0} p}\right]= & \left\{\left[\mathbf{v}_{\mathbf{0}} \otimes \mathbf{h}_{\mathbf{0} p}\right]\left[\mathbf{v}_{\mathbf{0}}{ }^{H} \otimes \mathbf{h}_{\mathbf{0} p}{ }^{H}\right] \sigma_{s}^{2}+\sigma_{n}^{2} \mathbf{I}\right\}\left[\mathbf{v}_{\mathbf{0}} \otimes \mathbf{h}_{\mathbf{0} p}\right] \\
= & {\left[\mathbf{v}_{\mathbf{0}} \otimes \mathbf{h}_{\mathbf{0} p}\right]\left[\mathbf{v}_{\mathbf{0}}{ }^{H} \mathbf{v}_{\mathbf{0}} \otimes \mathbf{h}_{\mathbf{0} p}{ }^{H} \mathbf{h}_{\mathbf{0} p}\right] \sigma_{s}^{2}+\sigma_{n}^{2}\left[\mathbf{v}_{\mathbf{0}}\right.} \\
& \left.\otimes \mathbf{h}_{\mathbf{0} p}\right]=\left[\mathbf{v}_{\mathbf{0}} \otimes \mathbf{h}_{\mathbf{0} p}\right]\left\{\mathbf{v}_{\mathbf{0}}{ }^{2} \mathbf{h}_{\mathbf{0} p}^{2} \sigma_{s}^{2}+\sigma_{n}^{2}\right\},
\end{aligned}
$$

where the quantity in brackets \{\} is simply the eigenvalue associated with this eigenvector. Then a maximization with respect to $\mathbf{e}$ is the eigenvector associated with the largest eigenvalue as given by:

$$
\hat{\mathbf{e}}=\frac{\mathbf{v} \otimes \mathbf{h}_{p}}{\sqrt{\left[\mathbf{v} \otimes \mathbf{h}_{p}\right]^{H}\left[\mathbf{v} \otimes \mathbf{h}_{p}\right]}}=\frac{\mathbf{v} \otimes \mathbf{h}_{p}}{\sqrt{\mathbf{v}^{H} \mathbf{v} \otimes \mathbf{h}_{p}^{H} \mathbf{h}_{p}}}=\frac{\mathbf{v}}{\sqrt{\mathbf{v}^{H} \mathbf{v}}} \otimes \hat{\mathbf{e}}_{p},
$$

where $\hat{\mathbf{e}}_{p}$ is the replica vector estimator for the pressure defined in Section II C and where properties 2 and 3 were used.

Replacing (A4) and (A1) in the generic Bartlett estimator (18), using the properties of the Kronecker product 2, 3 and 4 with subject to $\mathbf{e}_{p}^{H} \mathbf{e}_{p}=1$, the Bartlett estimator for the particle velocity model is given by:

$$
\begin{aligned}
P_{B} & =\frac{\mathbf{v}^{H}}{\sqrt{\mathbf{v}^{H} \mathbf{v}}} \otimes \hat{\mathbf{e}}_{p}^{H}\left\{\left[\mathbf{v}_{\mathbf{0}} \otimes \mathbf{h}_{\mathbf{0} p}\right]\left[\mathbf{v}_{\mathbf{0}}{ }^{H} \otimes \mathbf{h}_{\mathbf{0} p}{ }^{H}\right] \sigma_{s}^{2}+\sigma_{n}^{2} \mathbf{I}\right\} \frac{\mathbf{v}}{\sqrt{\mathbf{v}^{H} \mathbf{v}}} \\
& \otimes \frac{\hat{\mathbf{e}}_{p}}{\left(\mathbf{v}^{H} \mathbf{v}_{\mathbf{0}} \mathbf{v}_{\mathbf{0}}{ }^{H} \mathbf{v}\right) \otimes\left(\hat{\mathbf{e}}_{p}^{H} \mathbf{h}_{\mathbf{0} p} \mathbf{h}_{\mathbf{0}_{p}}{ }^{H} \hat{\mathbf{e}}_{p}\right) \sigma_{s}^{2}+\mathbf{v}^{H} \mathbf{v} \hat{\mathbf{e}}_{p}^{H} \hat{\mathbf{e}}_{p} \sigma_{n}^{2}} \\
\mathbf{v}^{H} \mathbf{v} & \\
& =\frac{\left[\mathbf{v}^{H} \mathbf{v}_{0}\right]^{2}}{\mathbf{v}^{H} \mathbf{v}} B_{p} \sigma_{s}^{2}+\sigma_{n}^{2} .
\end{aligned}
$$

Taking into account (24), one can conclude that the vector sensor estimator (with or without pressure) is proportional to the acoustic pressure estimator response, where the inner product $\mathbf{v}^{H} \mathbf{v}_{\mathbf{0}}$ is the constant of proportionality herein called directivity factor.

${ }^{1}$ A. Nehorai and E. Paldi, "Acoustic vector-sensor array processing," IEEE Trans. Signal Process. 42, 2481-2491 (1994).

${ }^{2}$ B. A. Cray and A. H. Nuttall, "Directivity factors for linear arrays of velocity sensors," J. Acoust. Soc. Am. 110, 324-331 (2001).

${ }^{3}$ J. Tabrikian, R. Shavit, and D. Rahamim, "An efficient vector sensor configuration for source localization," IEEE Signal Process. Lett. 11, 690-693 (2004).

${ }^{4}$ C. Wan, A. Kong, and C. Liu, "A comparative study of doa estimation using vector/gradient sensors," in Proceedings of Oceans 2006, Asia Pacific (2007), pp. 1-4.

${ }^{5}$ J. C. Shipps and B. M. Abraham, "The use of vector sensors for underwater port and waterway security," in Proceedings of the Sensors for Industry Conference, New Orleans, LA (2004), pp. 41-44.

${ }^{6}$ D. Lindwall, "Marine seismic surveys with vector acoustic sensors," in Proceedings of the Society of Exploration Geophysicists Annual Meeting,
New Orleans, LA (2006), pp. 1208-1212.

${ }^{7}$ Y. H. Wang, J. Q. Zhang, B. Hu, and J. He, "Hypercomplex model of acoustic vector sensor array with its application for the high resolution two dimensional direction of arrival estimation," in Proceedings of the $\mathrm{I}^{2}$ MTC 2008, IEEE International Instrumentation and Measurement Technology Conference, Victoria, Vancouver, Canada (2008), pp. 1-5.

${ }^{8}$ S. Miron, N. Le Bihan, and J. I. Mars, "Quaternion-music for vectorsensor array processing," IEEE Trans. Signal Process. 54, 1218-1229 (2006).

${ }^{9}$ A. Abdi, H. Guo, and P. Sutthiwan, "A new vector sensor receiver for underwater acoustic communication," in Proceedings of MTS/IEEE Oceans, Vancouver, BC, Canada (2007), pp. 1-10.

${ }^{10}$ A. Song, M. Badiey, P. Hursky, and A. Abdi, "Time reversal receivers for underwater acoustic communication using vector sensors," in Proceedings of MTS/IEEE Oceans, Quebec, Canada (2008), pp. 1-10.

${ }^{11} \mathrm{H}$. Peng and F. Li, "Geoacoustic inversion based on a vector hydrophone array," Chin. Phys. Lett. 24, 1997-1980 (2007).

${ }^{12}$ M. J. Hinich, "Maximum-likelihood signal processing for a vertical array," J. Acoust. Soc. Am. 54, 499-503 (1973).

${ }^{13} \mathrm{P}$. Gerstoft, "Inversion of seismoacoustic data using genetic algorithms and a posteriori probability distributions," J. Acoust. Soc. Am. 95, 770782 (1994)

${ }^{14}$ C. E. Lindsay and N. R. Chapman, "Matched-field inversion for geoacoustic model parameters using adaptive simulated annealing," IEEE J. Ocean. Eng. 18, 224-231 (1993).

${ }^{15}$ M. Porter, B. Abraham, M. Badiey, M. Buckingham, T. Folegot, P. Hursky, S. Jesus, K. Kim, B. Kraft, V. McDonald, C. deMoustier, J. Presig, S. Roy, M. Siderius, H. Song, and W. Yang, "The Makai experiment: Highfrequency acoustics," in Proceedings of the Eighth ECUA, Carvoeiro, Portugal (2006), edited by S. M. Jesus and O. C. Rodríguez, Vol. 1, pp. 9-18. ${ }^{16} \mathrm{P}$. Santos, P. Felisberto, and P. Hursky, "Source localization with vector sensor array during the Makai experiment," in Proceedings of the Second International Conference and Exhibition on Underwater Acoustic Measurements: Technologies and Results, pp. 985-990, Heraklion, Greece (2007).

${ }^{17}$ P. Santos, O. C. Rodríguez, P. Felisberto, and S. M. Jesus, "Geoacoustic matched-field inversion using a vertical vector sensor array," in Proceedings of the Third International Conference and Exhibition on Underwater Acoustic Measurements: Technologies and Results, Nafplion, Greece (2009), pp. 29-34.

${ }^{18}$ O. C. Rodríguez, "The TRACE and TRACEO ray tracing programs," http://www.siplab.fct.ualg.pt/models.shtml (Last viewed 6/7/10).

${ }^{19}$ F. B. Jensen, W. A. Kuperman, M. B. Porter, and H. Schmidt, Computational Ocean Acoustics, AIP Series in Modern Acoustics and Signal Processing (American Institute of Physics, Melville, New York, 1994), pp. $168-170$.

${ }^{20}$ Both between VSA elements and between sensors within each element.

${ }^{21}$ A. Tolstoy, Matched Field Processing for Underwater Acoustics (World Scientific, Singapore, 1993), pp. 14-22.

${ }^{22} \mathrm{H}$. Krim and M. Viberg, "Two decades of arraysignal processing research," IEEE Signal Process. Mag. 13, 67-94 (1996).

${ }^{23}$ C. Soares and S. M. Jesus, "Broadband matched-field processing: Coherent and incoherent approaches," J. Acoust. Soc. Am. 113, 2587-2598 (2003).

${ }^{24}$ H. Schmidt, "SAFARI: Seismo-Acoustic Fast Field Algorithm for RangeIndependent Environments, User's Guide," SACLANT Undersea Research Centre Report No. SR-113, La Spezia, Italy, 1988.

${ }^{25}$ C. H. Harrison and D. G. Simons, "Geoacoustic inversion of ambient noise: A simple method," J. Acoust. Soc. Am. 112, 1377-1387 (2002). 